\title{
Design Method for Developing a Product Recovery Management System based on Life Cycle Information
}

\author{
Jumyung Um' and Suk-Hwan Suh ${ }^{2 *}$ \\ 1 Institute for Manufacturing, University of Cambridge, 10 Charles Babbage Road, Cambridge CB3 OFS, United Kingdom \\ 2 Graduate School of Engineering Mastership, Pohang University of Science and Technology, 77, Cheongam-ro, Nam-gu, Pohang-si, Gyeongsangbuk-do, 790-784, South Korea \\ \# Corresponding Author / E-mail: shs@postech.ac.kr, TEL: +82-54-279-2196, FAX: +82-54-279-0138
}

KEYWORDS: Product recovery management system, UbiDMR, End-of-Life, Remanufacturing

\begin{abstract}
Due to concerns about environmental protection, product life cycle management for end-of-life has received increasing attention in many industrial sectors. To support these functions, crucial issues have been studied to realize a product recovery management system. Until the present time most research has been concerned with decision making under the assumption that all the relevant and accurate information about a product is available by some means. However, these pieces of research ended in technological attempts because of the development complexity of implementation using ubiquitous computing devices such as identification chips and embedded systems to get data from products. An efficient development method is necessary in order to overcome this limitation. In this paper we overview a generic architecture based on ubiquitous computing technology. This is followed by how to develop such an innovative system by proposing a systematic approach called ubiquitous information engineering. To show the effectiveness of the architecture and approach a prototype for remanufacturing an industrial product has been developed. Through development of the proposed approach enough functions can be derived to collect information from a product. The study shows that major factors influencing development complexity are found and that information standards support network development in end-of-life activities.
\end{abstract}

\section{NOMENCLATURE}

D2U = Device-to-UPLI

DISS $=$ Disassembly \& Inspection Support System

$\mathrm{ECU}=$ Engine Control Unit

IDEF $=$ Integration Definition for Function Modeling

IEEE $=$ Institute of Electrical and Electronics Engineers

PRMS $=$ Product Recovery Management System

RFID $=$ Radio Frequency IDentification

RTLS $=$ Real Time Locating System

$\mathrm{UbiDMR}=\mathrm{A}$ new paradigm of Design, Manufacturing, and Recycling via Ubiquitous computing

UPLI = Ubiquitous Product Lifecycle Information highway

WLAN $=$ Wireless Local Area Network

WSN $=$ Wireless Sensor Network

$\mathrm{XML}=$ eXtensible Markup Language

\section{Introduction}

Traditional manufacturers have ignored the existence of used products and considered them as landfill problems or incinerated them at their end-of-life stage. ${ }^{1}$ However, the responsibility of product manufacturers is now emphasized, and resource exhaustion and environmental pollution are now recognized as serious problems. The end-of-life industries including reuse, remanufacturing, and recycling operated by independent companies as well as original manufactures have been started with these motives. ${ }^{2}$ Some literature reports that the end-of-life industries can reduce environmental impact effectively. It has been found that remanufacturing reduces the environmental impact of end-of-life through the case study of photocopiers. ${ }^{3}$ The remanufacturing of engines in the United States of America provided significant reductions in energy and material consumption, air emission, and solid waste generation. ${ }^{4}$ For these reasons, remanufacturing is critical environmental criteria considered by sustainable manufacturing. ${ }^{5}$ To establish remanufacturing in a product market effective supporting systems are required. It is necessary to have efficient and flexible recycling systems, such as product value chains and company 
networks, ${ }^{6-8}$ which can handle various types of product in great quantities. ${ }^{9,10}$ To develop the system, a function which automatically makes recovery options based on part information and actual condition $^{11-13}$ is required. Product information is important in decision making at product end-of-life. However, most of the production information is lost after the product is sold and used by customers. ${ }^{14}$

To satisfy the lack of product information, a product recovery management system (PRMS) was developed. ${ }^{15}$ Product recovery management is formally defined as 'the management of all used and discarded products, components, and material for which a manufacturing company is legally, contractually or otherwise responsible'. ${ }^{16,17}$ PRMS is a system that can support decision making by gathering product information and finding the optimal solution. ${ }^{16,18}$ In PRMS, product information, such as how the product was originally manufactured, how the product has been used (repaired/maintained), and so on, is necessary. ${ }^{15}$ Looking at the current state-of-the-art of PRMS, the above will be hard to achieve unless new and innovative methods are developed. The characteristics of effective PRMS are obtaining the usage information of the product, transforming the usage information, and integrating the information for various stakeholders. Many information systems for end-of-life have been reported. ${ }^{19,20}$ It is hard for the PRMS supported by these systems to overcome the lack of product information and to create accurate and effective results. To deal successfully with these limitations, an innovative approach based on ubiquitous computing technology is needed. However, recent research has focused on proposing specific architectures and devices without discussing the development process for diverse products.

Recent issues for the manufacturing paradigm involve the traceability of individual products and whole product life cycle management because manufacturing is moving toward order-based production and closed-loop life cycles. To catch up with the trend, ubiquitous computing technology, which can provide information anytime anywhere such as automatic identification ${ }^{21}$ and real time information collection from small sensors, ${ }^{22,23}$ is employed in products and facilities in the life cycle. A new manufacturing paradigm based on ubiquitous computing technology is proposed, which is called UbiDMR (Design, Manufacture, and Recycling via Ubiquitous Computing Technology), whose main issue is the utilization of the entire product lifecycle information via ubiquitous computing technology for product design, manufacturing, and recycling. ${ }^{24}$ UbiDMR has the following characteristics, as presented in Fig. 1: it applies integrated manufacturing technology, information technology, and ubiquitous technology to the manufacturing domain; it covers manufacturing-related activities throughout the entire product lifecycle; it transparently collects and utilizes data on product and product-related contexts at individual item-level; and it supports real-time collaborative activities between stakeholders in a distributed environment. It also provides a conceptual framework and architecture for each service and shows that the following ubiquitous services were effective in various scenarios, namely: ubiquitous factory, ${ }^{25}$ product recovery management system, ${ }^{15}$ ubiquitous information infrastructure highway, ${ }^{26}$ computeraided ubiquitous system engineering, ${ }^{27}$ and ubiquitous technology based life cycle assessment. ${ }^{28}$ This paper proposes a service for the ubiquitous system called u-PRMS (PRMS based on ubiquitous computing technology), indicated by the dotted boxes in Fig. 1. To develop u-PRMS, ubiquitous information engineering is employed, which is the methodology for designing an information acquisition system via ubiquitous computing technology for the purpose of realizing UbiDMR.

In this paper we present the architecture for u-PRMS based on the ubiquitous product lifecycle information highway (UPLI), together with design considerations in Section 2 and information engineering for developing u-PRMS in Section 3. Based on the architecture and information engineering, the developed prototype system and a testing scenario are described in Section 4. Results and discussion issues from development experience are described in Section 5. The paper concludes with some remarks in Section 6.

\section{Overview of PRMS on Ubiquitous Computing Technology}

Basically the success of a remanufacturing application in any particular industry needs the analysis of technical, operational and market factors. ${ }^{29}$ The development of u-PRMS includes design considerations about the remanufacturing domain. Especially the effectiveness of u-PRMS also depends on the characteristics of the target products. Thus u-PRMS is not an effective system for all products. Roughly speaking, target products for which u-PRMS can be justified can be summarized as follows: high part price, long lifetime, frequent part replacement, and various usage patterns. These factors influence the boundary of the product group for application for uPRMS. To apply outside the boundary, efficient and effective systems should be developed. Thus design considerations were derived in order to make u-PRMS more suitable to small and short-life products

\subsection{Design Consideration for u-PRMS}

To reflect the UbiDMR paradigm in the concept of PRMS, our research team proposed the architecture for u-PRMS, and a data model used by this system. ${ }^{15}$ The system acquires usage information from sensors attached to the target product and makes decisions about product recovery. The architecture and functionality, together with technical implementation largely determine the usefulness of u-PRMS. In the remainder of this section, we discuss some of the considerations for u-PRMS in terms of infrastructure, stakeholders, and implementation.

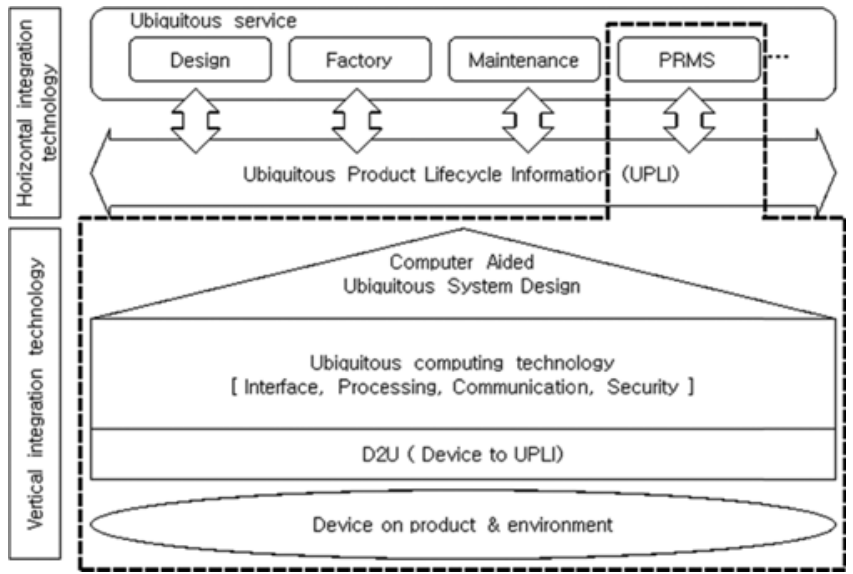

Fig. 1 Enabling technology for UbiDMR ${ }^{24}$ 
The perspective of infrastructure

1) Design consideration \#1 - Information access infrastructure: To obtain product information from various databases of stakeholders the PRMS infrastructure must be established.

2) Design consideration $\# 2$ - Internet interface: PRMS should provide an interface to access the databases of every stakeholder in the product lifestyle via the internet.

3) Design consideration \#3 - XML (eXtensible Markup Language) and ontology support: To enable querying, updating, and integrating target product data in PRMS, the information should be delivered as the data format representing relevant expertise extensively used by each stakeholder.

4) Design consideration \#4 - Use of standardized information: The products dealt with by PRMS should not be limited by physical area and information domain. For interoperability and domain-free usage PRMS should be able to interface with international standards. ${ }^{30-32}$

5) Design consideration \#5 - Integration capability: Information obtained from various sources, such as databases of stakeholders and embedded devices, needs to be combined to produce high-level information for remanufacturing and recycling. This is a key function determining the performance of PRMS.

6) Design consideration \#6 - Comprehensiveness of information contents: Information provided by PRMS should be comprehensive so that recycling and remanufacturing can be performed accurately and optimally.

The perspective of implementation

7) Design consideration \#7 - Comprehensiveness of information gathering ubiquitous device: PRMS should be able to read information from various devices embedded in the product, including radio frequency identification (RFID), small sensors, etc.

8) Design consideration \#8 - Multi-tasking capability: Tasks for assembly and disassembly during remanufacturing or recycling require information about multiple components. For the sake of task efficiency PRMS should have multi-tasking capability to provide multiple product information for users.
9) Design consideration \#9 - Generation of disassembly information: If disassembly information is not available from the product information, then PRMS should generate the disassembly sequence to replace the bad part(s) during remanufacturing. The sequence can be represented by AND/OR graph and transition matrix. ${ }^{33}$

10) Design consideration $\# 10$ - Variety of human-computer interface devices: Human operators may access PRMS via various devices in various situations

The stakeholder perspective

11) Design consideration \#11 - Target products: Considering the target product group is the first step in designing PRMS. This determines the data model of information gathered from stakeholders and the recovery decision algorithm.

12) Design consideration \#12 - Knowledge-based functionality: The purpose of PRMS is, in essence, to provide sufficient information so that the user can make the best decisions. This capability will be very useful for non-expert users. Just to support recovery decisions, this functionality is not mandatory.

13) Design consideration \#13 - Virtual simulation: Like computeraided manufacturing software, PRMS should display virtual operations to identify any possible errors before execution by remanufacturing.

14) Design consideration \#14 - Feedback to the original manufacturer: To close the product lifecycle loop, all the relevant information observed in the recycling process via PRMS should be fed back to the original manufacturer for redesigning the original product. Note that this is also a crucial factor for eco-design considering the environmental aspect in the product development stage. ${ }^{34}$

\subsection{Generic Architecture}

The generic architecture is derived from the design considerations described above. These considerations are utilized to design the functions and the architecture of u-PRMS. As shown in Fig. 2, the

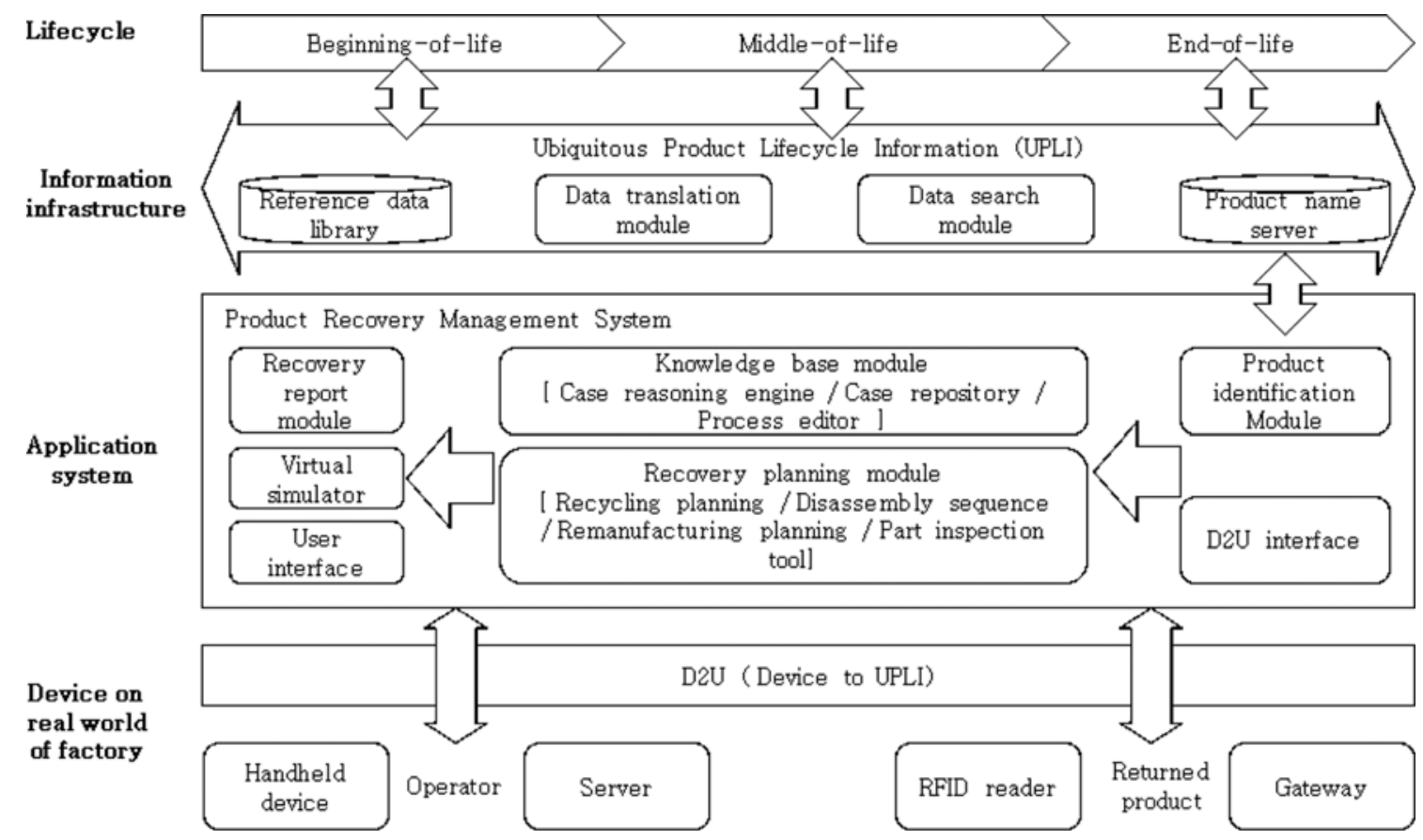

Fig. 2 Generic architecture for u-PRMS ${ }^{15}$ 
generic architecture is composed of four layers: life cycle, information infrastructure, application system, usage environment in the real world. The lifecycle layer means the status of the product from the perspective of lifecycle: beginning-of-life, middle-of-life, and end-of-life. The second layer, information infrastructure, is the infrastructure where the information is transformed, exchanged, and retrieved by the various stakeholders. The UPLI (Ubiquitous Product Lifecycle Information) network explained below belongs to this layer. The third layer, application system, consists of the various systems used by the stakeholder for the purpose of lifecycle activity by interfacing with the information infrastructure. PRMS belongs to this layer. The fourth layer, real world device, is used to acquire/aggregate the product information via sensors, RFID, sensor network. Since the PRMS is closely related to the information infrastructure and application layers, the details of the modules in these two layers are given below.

\section{Information infrastructure (UPLI)}

1) Product name server: This server stores the information about linking and binding the product identification and its corresponding internet protocol address indicating stakeholder databases. This explains why PRMS is required to have an interfacing capability with the UPLI based on design considerations \#1 and \#2 given in Section 2.1 .

2) Data search module: This module searches product information from the databases on the internet with the internet protocol address given by the product name server. For the sake of speed of PRMS, this module should search in a simultaneous manner. This complies with design consideration $\# 8$.

3) Data translation module: In general, the data models including terminology and information hierarchy obtained from databases of various stakeholders are not consistent and so require translation. For this purpose, mapping the local data into international standard data (complies with Design consideration \#4) via ontology representation language such as extensible markup language and web ontology language is required, complies with Design consideration \#3.

4) Reference data library: This library is a database storing the mapping relationships with local ontology and the international standard ontology. It should be noted that the reference data library and product name server should be synchronized in such a way that if an internet protocol address for a new database of a stakeholder is added to the product name server, then a local ontology corresponding to the uniform resource locator should be added to the reference data library (complies with Design consideration \#5). The international standards for reference data library can be found in ISO 10303-239. ${ }^{32}$

\section{Product Recovery Management System (PRMS)}

5) Product identification module: This module requests product information from the information infrastructure for the product identification and stores it in the PRMS database. To increase the performance of this module, the module should connect to a number of databases simultaneously (complies with Design consideration \#6).

6) D2U (Device-to-UPLI) interface: The D2U interface module converts information from the D2U to the information for the PRMS modules. Thus, interfacing capability with various ubiquitous computing devices is required, as described in Design consideration \#7.
7) PRMS database: This database stores all product information related to its product recovery such as computer-aided design, bill of material, usage information, previous remanufacturing cases, etc. This is necessary to satisfy requirements mentioned in many design considerations, such as \#1, \#4, and \#6.

8) Knowledge base module: This module uses previous cases to improve the efficiency and accuracy of recovery decisions. This complies with Design consideration \#12.

9) Recovery planning module: This is the main function of PRMS that the user (e.g., an operator in a remanufacturing facility) can appreciate. PRMS supports users in the process of remanufacturing. Each of these modules should interface with the knowledge base module to provide useful information to the user. In this way, information can be provided for disassembly, especially for complex products (complies with Design considerations \#9 and \#11).

10) Recovery report module: This is for the closed-loop information interface between the remanufacturer and manufacturer. This is a key aspect that is emphasized in this paper as a means for improving the design of the next product generation. To this end, this module reports information at the end-of-life stage including the recovery cost and option used, problems occurring at the remanufacturing stage, and general statistics, such as damage statistics and component failure rates. This complies with Design consideration \#14.

11) Virtual simulator: This module supports users by showing the planned operation via graphic simulation. This module complies with Design consideration \#13.

12) User interface: This interface provides an interface between PRMS and user devices. This module complies with Design consideration \#10.

\section{Ubiquitous Information Engineering}

The customization to target product and adaptation of the generic uPRMS architecture is necessary because the characteristics of the product influence the implementation architecture. This paper shows how the generic u-PRMS architecture can be applied to a specific product taking a starter motor as an example. An emphasis is given to developing a structured method to design the information acquisition method called ubiquitous information engineering, the vertical column technology in the technology map of UbiDMR of Fig. 1 for u-PRMS.

Ubiquitous information engineering can be defined as a systematic approach to designing a ubiquitous information acquisition system, which is a set of all components acquiring the information needed for ubiquitous service from Product / Process / Resource / Environment / User / Organization of the real world over the whole product lifecycle by various kinds of method in order to provide information to the stakeholders. Ubiquitous information engineering is also an engineering approach for information acquisition methods in the UbiDMR environment and focuses on how to acquire the information to be used in the ubiquitous service of various stakeholders based on real field data. ${ }^{35}$ The ubiquitous service is the application system that is provided by stakeholders in the product life cycle based on ubiquitous computing technology internally or externally. As summarized in Fig. 3 , the ubiquitous information engineering methodology consists of four 
stages: Objective and use case definition, Requirement analysis, System design, and Validation and verification.

Note that the stages of this methodology are derived from system engineering processes used for designing complex systems such as aircraft, military equipment, and aerospace equipment. ${ }^{36}$ Ubiquitous information engineering is derived from the system engineering process where the outputs are the documents of functions and processes in the developed system. The function and process of a ubiquitous information acquisition system are determined by ubiquitous service and the activity of product life cycle. This section describes the processes.

Since the purpose of the proposed system is to collect the product information required for the ubiquitous service, the service provided target product should be determined. To make a decision, the product type and scope of the service should be known. Standardized systems for product classification ${ }^{37}$ are used for the purpose of defining the scope of services because the products are grouped with considerations about the industrial characteristics of production, logistics, usage, and disposal.

When the target product is determined, the service provider, its service, the client of the service should be defined. A service blueprint ${ }^{38}$ represents the relationship between its provider and its client as well as the original service process. In the case of u-PRMS, its service is a support service of remanufacturing guidelines which the manufacturer provides to remanufacturing factories.

\subsection{Stage 1: Objective and Use Case Definition}

Objective and use case definition is the stage of defining the purpose and the usage scenario of the information acquisition system which will be developed. Objective definition means defining the purpose of information acquisition. The objective tree method $^{39}$ or quality function deployment ${ }^{40}$ can be used for classifying objectives into sub-objectives and evaluating the importance of the sub-objectives. For example, the major objective of the information acquisition system for u-PRMS is to decide whether each part is reused or recycled. To do this, the sub-objectives are information collection, information integration, and identification of part status.

The use case definition involves deriving to-be scenarios including the activities and information flow of the modules of the information acquisition system. For example, a possible scenario of u-PRMS is to collect part status and maintenance history in the usage stage and to make a decision on the remanufacturing based on collected information when a product is disposed of. The information output from this stage concerns objectives and use case of the information acquisition system.

\subsection{Stage 2: Requirement Analysis}

The second stage is the activity to derive design considerations and functions and to define data requirements in accordance with the objectives and use cases defined in the previous stage. Data requirement describes what data should be captured by the information acquisition system. Data requirements include the format, range, memory size, and data collection frequency of the product information required for the service.

Design consideration means the constraints for designing the information acquisition system. Design considerations are divided into the considerations required by the service provider and client, considerations of information infrastructure exchanging product information, and considerations of implementing ubiquitous computing devices in the real world such as contactless identification system (RFID: Radio Frequency Identification) and location tracking system (RTLS: Real Time Locating System). In the case of the u-PRMS applied to vehicles, it is possible to exchange data between the D2U in its engine and main server regardless of the location of the position of vehicles.

Functional derivation involves deriving necessary functions in order to carry out the activities in the usage scenario. At this stage functions for collecting large amounts of data are required to accommodate the

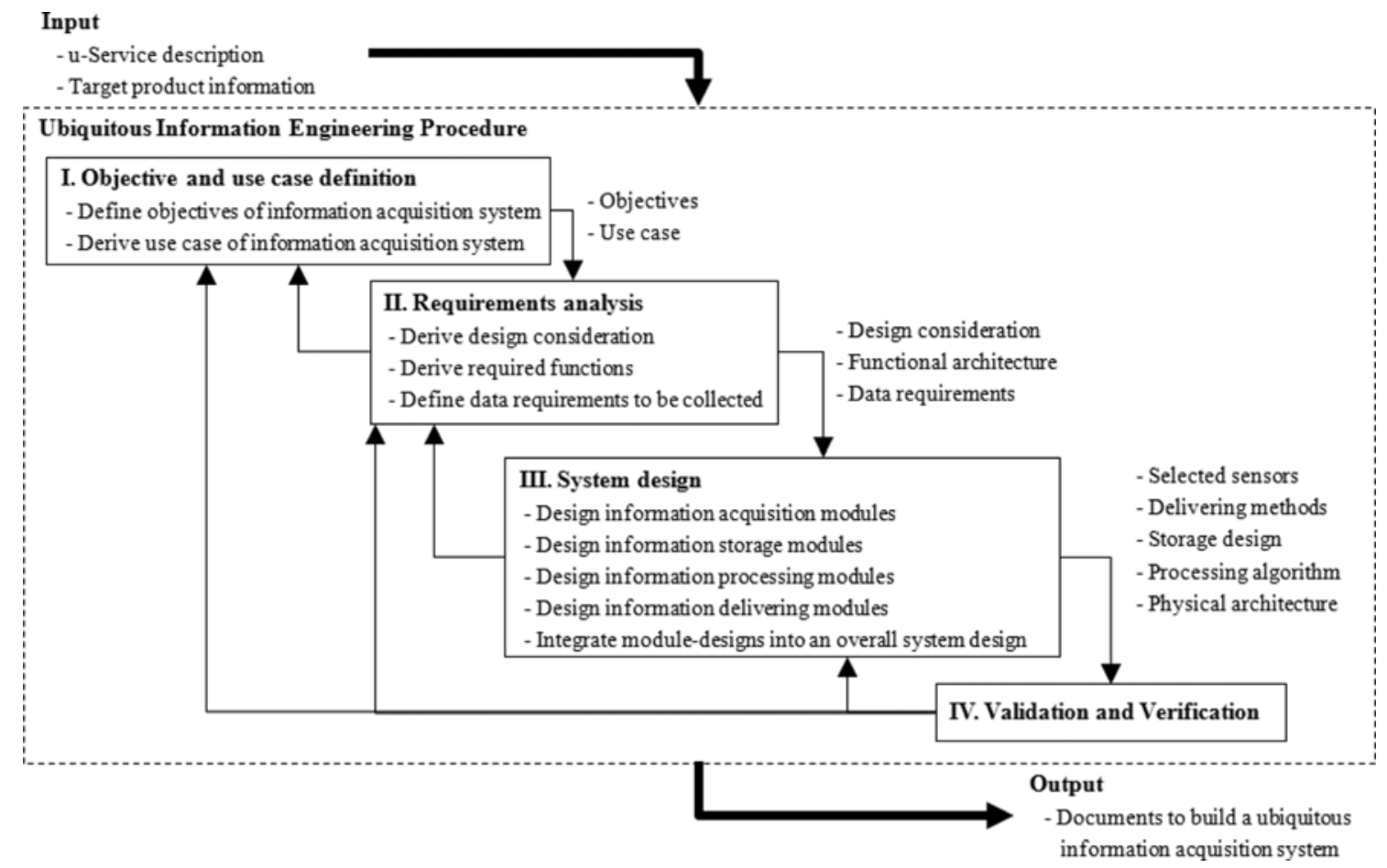

Fig. 3 Procedure of ubiquitous information engineering 
design considerations. The IDEF0 (Integration Definition for Function Modeling $)^{36}$ diagram is useful for this functional derivation. In the case of a D2U of u-PRMS, sensor data collection and wireless communication are the functions that the D2U should carry out.

\subsection{Stage 3: System Design}

In the system design, the modules composing the information acquisition system to satisfy the requirements analyzed in the previous stage are designed. The composition modules are classified into an acquisition module, delivery module, storage module, and processing module from the perspective of the role in usage scenarios. The acquisition module is a module that collects real world data such as that of products, the environment, humans, etc. The design criteria for the information acquisition module are sensor type and attachment location of the sensor. Selecting the sensor type means finding a sensor that suits the physical phenomena which should be detected. Determining the attachment location means finding parts and locations attachable for the selected sensor. For example, $\mathrm{X}$ and $\mathrm{Y}$ acceleration sensors are attached to the surface of the housing to acquire the vibration data of a starter motor.

The delivery module is a module that transmits the data captured by an acquisition module into data storage such as a memory or a database. The design criteria are the communication protocol, which is suitable for the situation of a target product, and the communication device with selected protocol. When the sensor data inside an engine are collected, wired cables are suitable because the distance between the data collection system and sensor is short. However, if the installation of cables for a sensor is difficult, then a wireless sensor using a short-distance communication protocol (e.g., IEEE 802.15.4) or wireless local area network (WLAN) access point is selected for the communication device.

The storage module is a module for storing the collected data. The design criteria are the location type and the database structure of the storage. The location types of storage are largely small-size memory devices inside the product and an information system outside the product. If the storage capability of the product has not enough space because the product information is too great, then external database is effective and efficient. For example, in the case of implementing the usage information database for construction machinery, a customer relationship management server or maintenance information system is a location available for installation of the storage module. Designing a database structure involves determining the primary key and table criteria.

The processing module uses an algorithm deriving the information required for service by processing the collected data. The design criteria are the information processing algorithm, the procedure of information processing. The operation time and number of operations can be calculated via the sensor data of the starter motor. The procedure of information processing is necessary because various algorithms are conducted sequentially along the information flow. For example, the failure rate is calculated with the operation time derived from vibration sensor data. To do this, noise reduction, filtering, operation time calculation, and lifetime algorithms are typically used sequentially.

The implementation architecture shows acquisition / delivery / storage / processing modules derived from this system design, duplications or additional interfaces between modules, and the whole information flow. This architecture is also an intermediate result of this design process. In the case of u-PRMS for a starter motor D2U, usage data collection system, PRMS, and handheld devices are the components of u-PRMS. Details about these components are given in section 4 .

\subsection{Stage 4: Verification and Validation}

Stage 4 examines whether or not the architecture designed in stage 3 satisfies the requirements of the information acquisition system. The verification and validation steps examine the performance of the operating developed prototype with a demonstration scenario derived from the usage scenario and compensation for the defects that occurred during the operation. The examination process is to compare the activities of to-be scenarios with the activities of demonstration scenarios and to derive the advantage of the prototype. For example, if a device adopting a low-speed and short-distance communication protocol (e.g., IEEE 802.15.4) is too slow to transmit the sensor data of part of the engine, then the designer goes back to stage 2 and should add new constraints to the design consideration of the information infrastructure. To reflect a new constraint, a communication device may be changed to wireless local area network (WLAN) for the delivery module in stage 3 . After it is validated and verified in the next stage and redesigned, it becomes the final architecture.

\section{Prototype Implementation}

To verify the validity and effectiveness of the developed methods, a u-PRMS prototype was developed. The product was chosen based on the considerations: The salvage process of the machinery is almost a manual operation requiring high expertise while the remanufacturing market is well developed. Based on this we selected a part of engine used in construction machinery, a high demand item in the remanufacturing market. The salvage process can be improved by the remanufacturing guidelines for individual machinery provided by uPRMS. ${ }^{41}$ The prototype gives the operator the information required for remanufacturing these parts.

The service for u-PRMS is the remanufacturing service of construction machinery. This service is based on real company A, which remanufactures construction machinery. Company A collects used machines by paying some price through a dealer, and remanufactures them. u-PRMS supports the remanufacturing operators by providing the remanufacturing guidelines for individual products. The information required for this service includes the final status of parts, disassembly instructions, and environmental regulations for the products. The scope of the prototype is the information system acquiring the information which is called ' $\mathrm{u}$-PRMS for a starter motor'. This section describes the development process of u-PRMS for the starter motor.

\subsection{Objective and Use Case Definition}

The remanufacturing growth of this machinery is limited now. Refurbishing core parts with high quality is difficult because a company requires enough experts to conduct manual technical processes. The survey shows that only companies occupying more than $5 \%$ of the market operate remanufacturing services for their own products. In order to overcome this limitation, expert knowledge is utilized by gathering usage data and binding it to previous cases. Therefore the main objective of the prototype is defined as gathering 
usage data. It also has 5 sub-objectives: identifying individual products, checking usage conditions, checking part condition, collecting part history, and delivering the collected data.

The following usage scenario shows the process in which u-PRMS collects the information and provides it to the operator. The use case of u-PRMS is divided into the middle-of-life phase and the end-of-life phase, as shown in Fig. 4. Modules in the scenario such as D2U, PRMS, and handheld devices comprise the modules for the generic architecture of u-PRMS. In middle-of-life, the manufacturer provides maintenance services and remanufacturing services to its customers by monitoring products through embedded devices, called D2U. D2U collects sensor data and the data of an engine control unit (ECU) whenever abnormal signals occur due to a broken part. A wide area network device in the machine enables the D2U to connect with the manufacturer in spite of machine movement. The manufacturer establishes the database system for collecting usage information including sensor data as well as product data management information such as bill of materials, assembly structure, environmental regulation and maintenance history. In end-of-life one machine is disposed of and returned to a remanufacturing factory. The operator identifies the returned part of the machine and requests the product information from PRMS. PRMS collects the information from the manufacturer through UPLI and provides PRMS information including failure-prone parts and disassembly instructions to operators. To realize this scenario, the specification of data collected by u-PRMS, the functional requirements of the data collection and the design considerations to be implemented in a product are derived in the next section.

\subsection{Requirement Analysis}

Requirement analysis is the stage for analyzing the requirements of modules in the usage scenario derived from the use case definition. The analysis is carried out according to the following questions: What information should be acquired from construction machinery and the information systems managed by its manufacturer (Data requirements)? What are the considerations from the perspective of the construction yard, manufacturer, and remanufacturing factory (Design consideration)? What

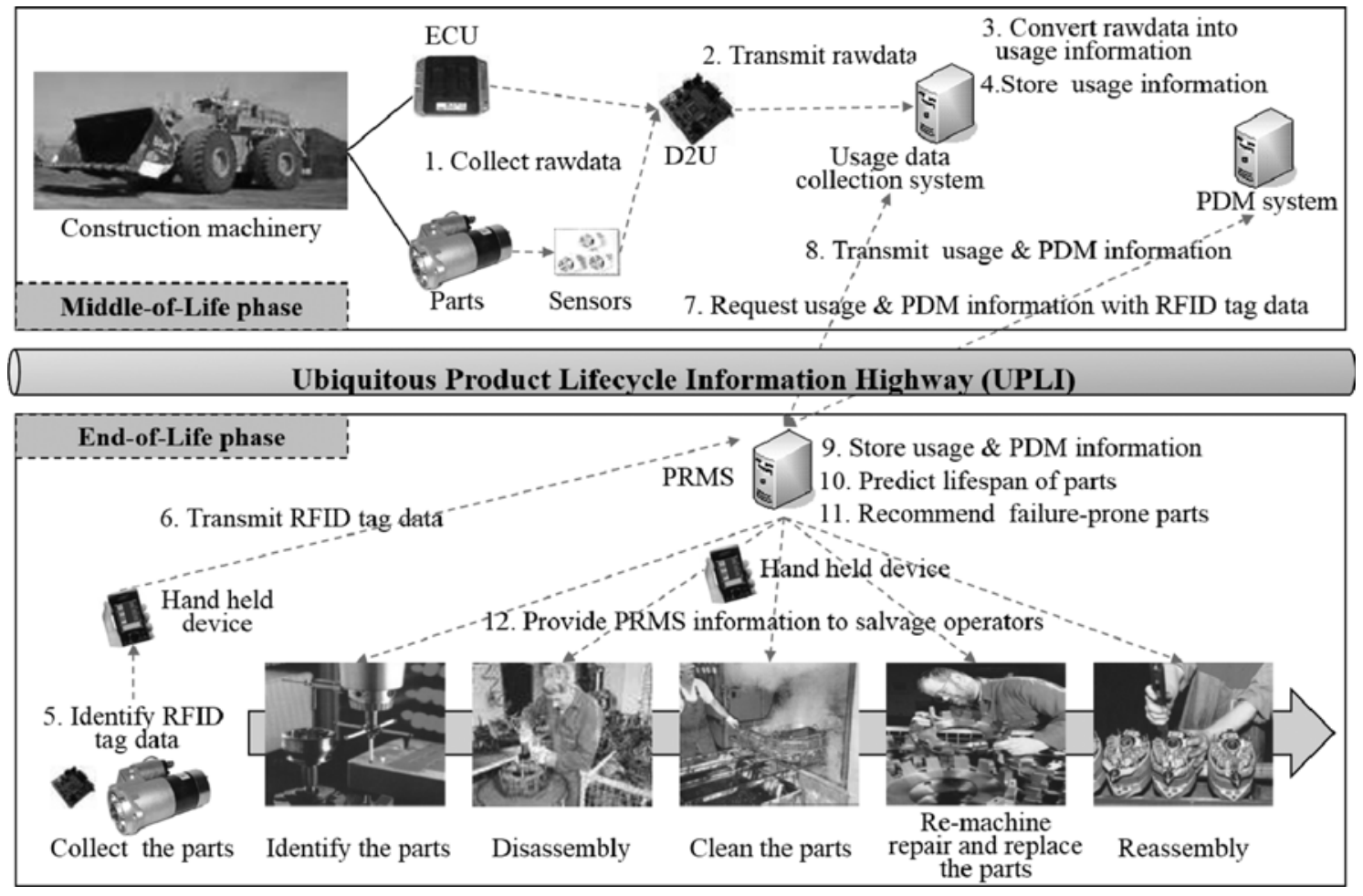

Fig. 4 Usage scenario of the u-PRMS for starter motor

Table 1 Data requirements of u-PRMS for starter motor

\begin{tabular}{|c|c|c|c|c|c|c|}
\hline Classification & Name & Type & Range & Format & Memory size & Data collection frequency \\
\hline \multirow{2}{*}{$\begin{array}{c}\text { Product } \\
\text { identification }\end{array}$} & Product name & Text & - & String & 512 Byte & When product is made \\
\hline & Product ID & Text & - & String & 512 Byte & When product is made \\
\hline \multirow{5}{*}{ Part condition } & Run time & Digital & $157,680,000$ & Decimal & 4 Byte & Every minute \\
\hline & Number of start, Stop & Digital & 14,600 & Decimal & 2 Byte & When motor starts to operate \\
\hline & Vibration & Digital & $-30 \sim 30 \mathrm{~g}$ & Real, Array & $5 \mathrm{MB}$ & $30 \mathrm{~g}>$ \\
\hline & Temperature & Digital & $-25 \sim 200^{\circ} \mathrm{C}$ & Real, Array & $5 \mathrm{MB}$ & $150^{\circ} \mathrm{C}>$ \\
\hline & Humidity & Digital & $0 \% \sim 100 \%$ & Real, Array & $5 \mathrm{MB}$ & $50 \%>$ \\
\hline \multirow{2}{*}{$\begin{array}{c}\text { Maintenance } \\
\text { history }\end{array}$} & Broken part & Text & 10 parts & STEP (AP239) & 512 Byte & When product is broken \\
\hline & Replaced part & Text & 10 parts & STEP (AP239) & 512 Byte & When product is repaired \\
\hline \multicolumn{2}{|c|}{ Assembly structure } & Text & 10 parts & STEP (AP203) & $1 \mathrm{MB}$ & When product is made \\
\hline \multicolumn{2}{|c|}{ Environment regulation } & Text & 500 regulations & String, Array & $1 \mathrm{~KB}$ & When product is made \\
\hline
\end{tabular}


are the functions necessary for modules of u-PRMS (Functional design)?

Three modules of the u-PRMS for a starter motor acquire data from a real world situation. Table 1 shows the data requirements. D2U collects temperature, humidity, and vibration sensor data from the starter motor while in use. Bearings and electronic parts as well as insulating materials are weak against high temperature. Bearings and shafts are corroded by high moisture. Shaft misalignment in the center of the motor causes motor vibration and the reduction of lifetime for the bearing. Besides the three sensor data elements, the operation time and number of uses of the starter motor are derived from the vibration profile. Product data management stores the assembly information written in ISO $10303-224^{42}$ and regulations relevant to construction machinery. It also stores the time based maintenance and maintenance history given by the maintainer of the machinery. The maintenance history includes dates, broken parts, maintenance method, and replaced parts. A handheld device equipped with an RFID reader finds the product model with 8 digits of hex characters stored in the radio frequency identification tag attached to the motor.

The next step in the design is to accommodate the voices of the stakeholders participating in the service activities, information infrastructure, and physical environment where the prototype is implemented. From the perspective of stakeholders, it is necessary for the exchange of individual product information items between the manufacturer and remanufacturer who are the stakeholders of the service for u-PRMS for a starter motor. The requirement for the information infrastructure is that it should be possible to connect between a construction yard and a manufacturer of machinery via long range wireless communication. The requirements of implementation are that the size of sensors and memories should be small enough to be installed in the engine, remanufacturing operators can briefly identify a number of starter motors, and handheld devices can provide customized remanufacturing guidelines to the operators such as inspector, disassembly operator, recycler, etc.

The final step in this stage is to derive what functions are needed to provide the service described in the usage scenario. The functions, which each module needs to acquire the data required for product recovery in the usage scenario of u-PRMS for a starter motor, are derived. D2U collects sensor data and transmits it via wireless communication. The usage data collection system processes the data

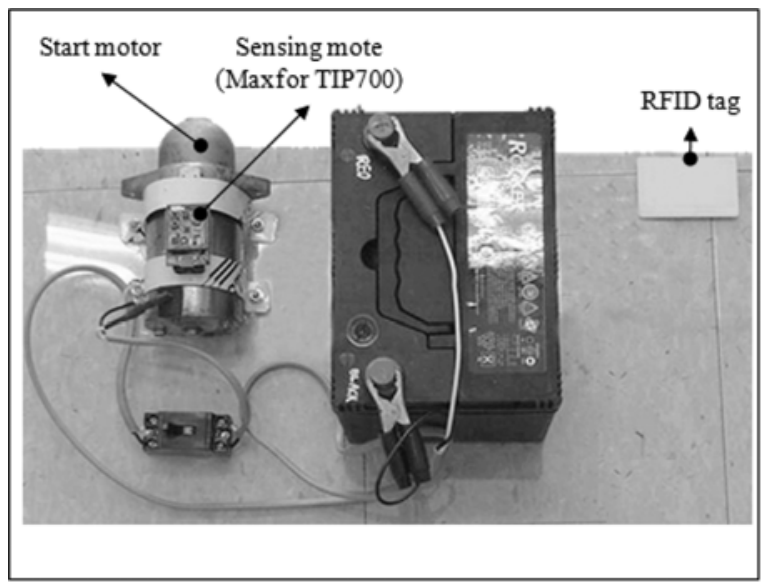

(a) Starting motor and sensing mote from wireless communication and stores usage data. Product data management provides the assembly structure, environmental regulations, and maintenance history. PRMS generates and transmits remanufacturing guidelines to operators and stores remanufacturing history. The handheld device identifies the returned motor and displays remanufacturing guidelines. The detail design carried out based on these functions is presented in section 4.3.The second stage is the activity to derive design considerations and functions and to

\subsection{System Design}

System design carries out the detail design of D2U, usage data collection system, PRMS and DISS (Disassembly \& inspection support system) installed in a handheld device to be used by operators as a kind of expert system. Each module is composed of acquisition module, delivery module, storage module, and processing module, as described in section 3.3

\subsubsection{D2U (Device to UPLI) Module}

$\mathrm{D} 2 \mathrm{U}$ is a device that collects sensor data and transmits it via wireless communication. The design criteria are sensor type and attachment location of the sensor because the collection sensor data belong to the function of the acquisition module. The sensors of D2U are humidity sensors, temperature sensors, and accelerometers. Small sensors are selected due to space limitations in the engine.

Transmitting data via wireless communication is the function of the delivery module. Its design criteria are communication protocol and the communication device. D2U uses two types of communication device. The first device is a wireless sensor network (WSN) mote to exchange data between the sensor mote and gateway system via low power and short range communication. It is a small communication device using personal area network protocol (IEEE 802.15.4). The second device uses a communication module for wide area network to exchange data between the gateway system and the usage data collection system. D2U is composed of a starter motor and sensors and an embedded gateway system based on two functions as shown in Fig. 5.

\subsubsection{Usage Data Collection System Module}

The usage data collection system processes the data from wireless communication and stores usage data. The design criteria are the information processing algorithm and procedure of information

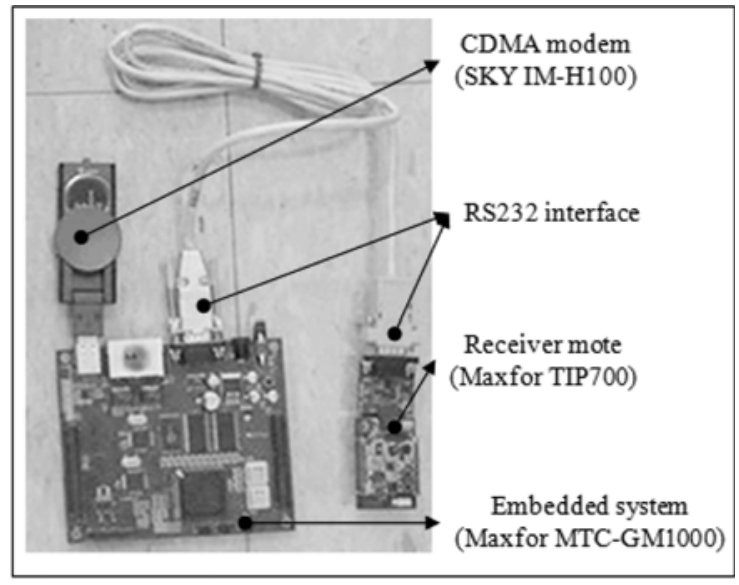

(b) Embedded gateway system

Fig. 5 Implementation of D2U prototype 
processing because processing the data is a function of the processing module. The information processing algorithms of the usage data collection system are a packet processing algorithm, noise reduction algorithm, and a filtering algorithm which removes outliers.

The design criteria are location of storage and database structure for the storage because usage data collection belongs to the function of the storage module. The external database is determined as a storage module due to concerns about the amount of sensor data from the

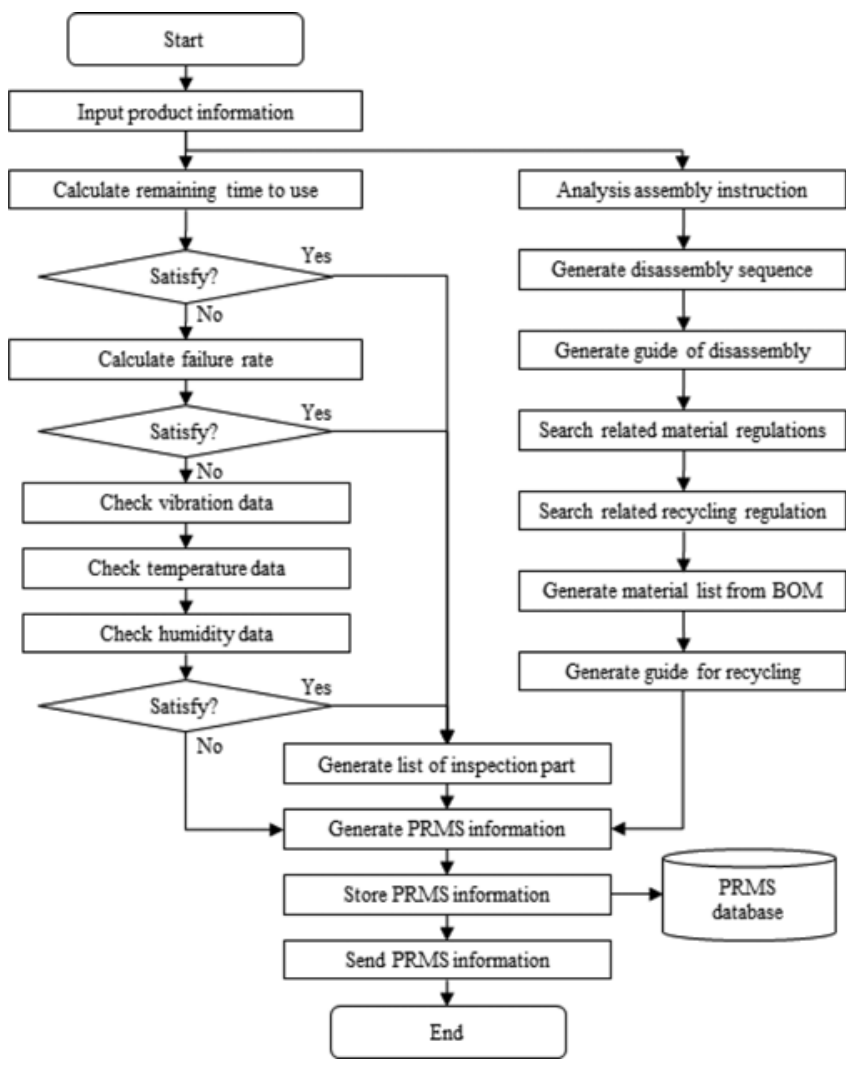

Fig. 6 Operation procedure of information processing module motor over ten years. The database is operated by the manufacturer.

\subsubsection{PRMS (Product Recovery Management System) Module}

PRMS generates and transmits the remanufacturing guidelines to operators and stores remanufacturing history. The generated guideline, which is a function of the processing module, consists of lifetime prediction/failure rate calculation/part status evaluation/disassembly sequence/regulation search algorithm. Fig. 6 shows the procedure for information processing. If the product information is received, then one procedure includes lifetime, failure rate, and product status, while the other is composed of generating guidelines for disassembly and recycling. Two results from two procedures are combined into the PRMS information, which will be sent to operators and stored in the PRMS database. The PRMS information is the remanufacturing guideline, which supports operators.

The PRMS is implemented based on three functions, as shown in Fig. 7, which consist of an information processing module, operation communication module, which is a delivery module, and a PRMS database manager that is a storage module.

\subsubsection{DISS (Disassembly \& Inspection Support System) Module}

DISS identifies motors returned and displays the remanufacturing guidelines. Identifying returned motors is a function of the acquisition module. An RFID reader identifying individual RFID tags is attached to the handheld device for realizing the function of acquisition module.

Operation viewers to display the remanufacturing guidelines are composed of a product identifier viewer, enabling all operators to identify each part, usage data viewer, failure-prone parts viewer, which provides disorder sensor data and broken parts to inspection operator, disassembly and assembly viewer, and regulation viewer showing the recycling guideline relevant to parts and operator reporter, as shown in Fig. 8. The technical issue of DISS implementation is the design consideration of selecting the user interface device of salvage operators. The device convenience depends on the type of workspace.

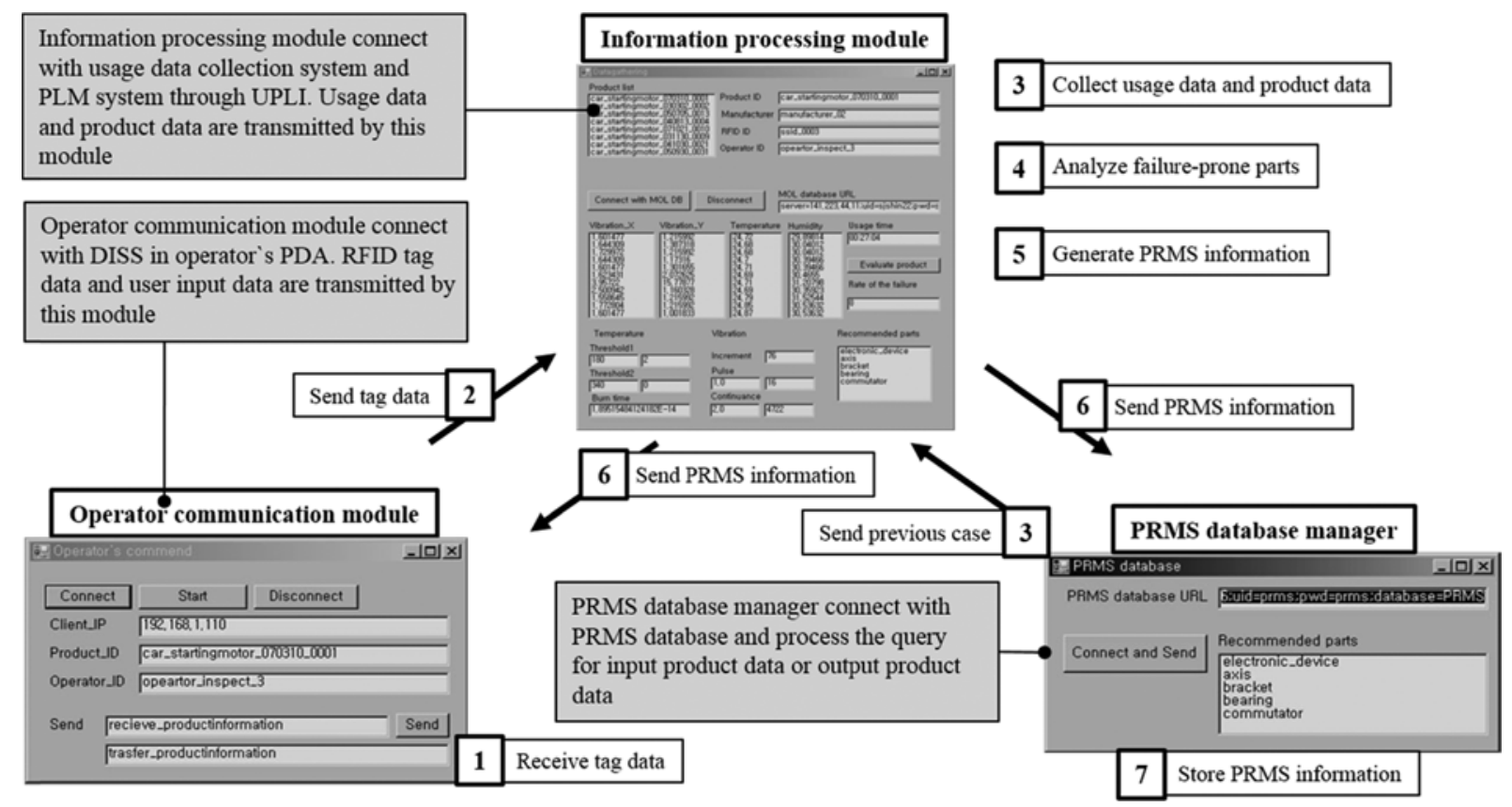

Fig. 7 Operation procedure of PRMS module 
Procedures and details of the operation views are described in the demonstration scenario in section 4.4.

\subsubsection{Implementation Architecture}

An implementation architecture is derived from the four mod gules described in sections 4.3.1 to 4.3.4, as shown in Fig. 9. These four modules are the minimum elements essentially required for realizing a genuine u-PRMS. This architecture follows the generic architecture illustrated in Fig. 2. The information infrastructure layer is UPLI, and the application system layer consists of a usage data collection system and PRMS module. The real world layer consists of the D2U module and the DISS module. The left side contains the modules operating
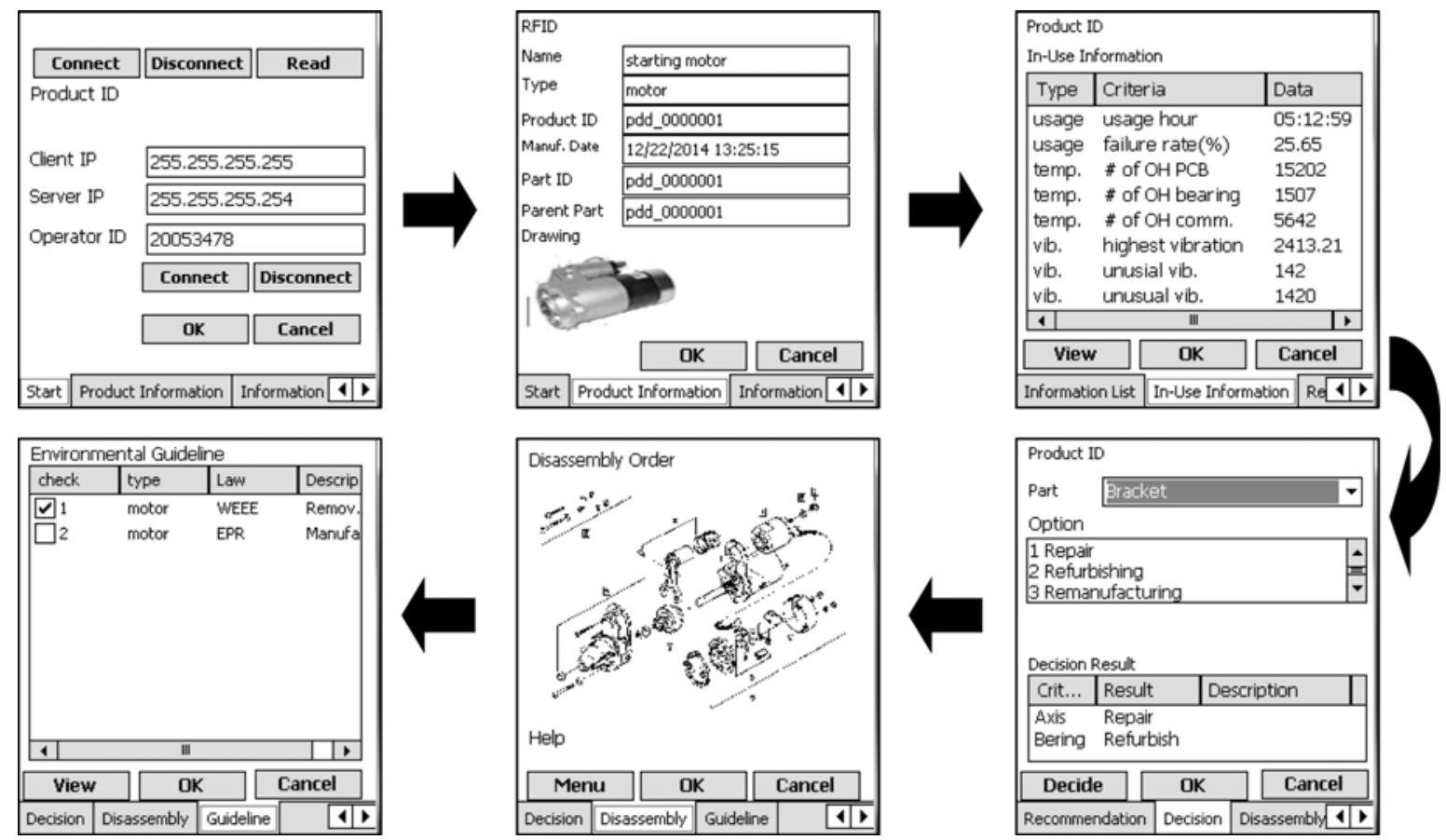

Fig. 8 DISS screens during demonstration scenario

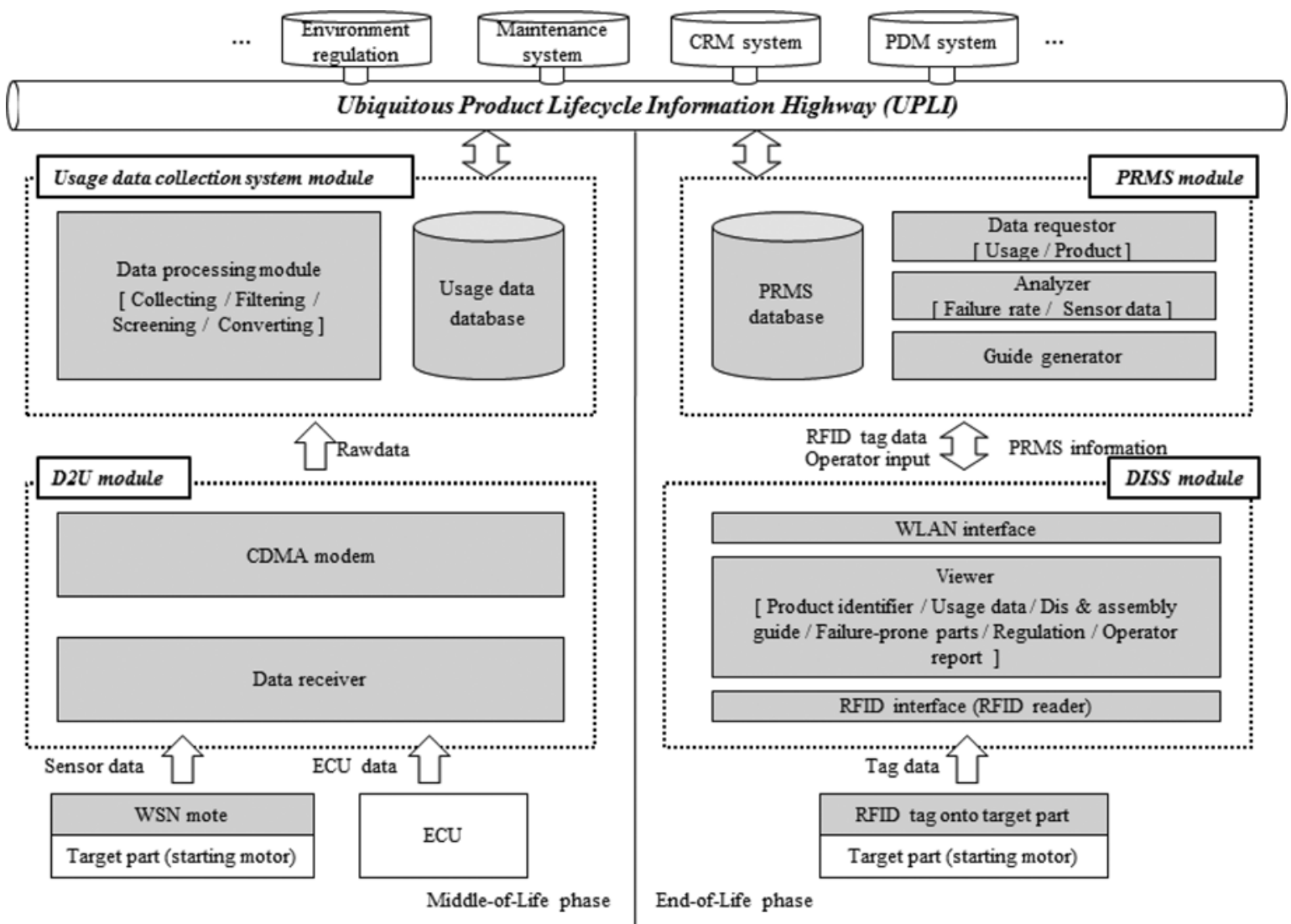

Fig. 9 Implementation architecture of u-PRMS for starter motor 
during middle-of-life, while the right side shows modules operating at end-of-life. The middle-of-life information flow starts in WSN mote in the D2U module, passed packet converter and four data processing functions, and ends in the usage data database. The end-of-life information flow starts from the RFID interface in DISS module, goes up to two request modules in the PRMS module, passes two analyzers and one guide generator, and ends in viewers in the DISS module. The modules in the middle-of-life phase communicate with the modules in the end-of-life phase via UPLI. UPLI is out of scope of this prototype.

\subsection{Demonstration Scenario}

To show how the developed system works, the demonstration scenario starts from the perspective of the DISS and of environmental impact with an assumption. The operation of DISS is illustrated in Fig. 8. A remanufacturing operator pushes the connect button on his/her handheld device, and the handheld device connects with PRMS. When a machine is discarded and transferred to the remanufacturing factory, the operator contacts the RFID reader of the handheld device via the RFID tag attached to the motor. The handheld device recognizes the serial number stored in the RFID tag and displays it. When the operator pushes the $\mathrm{OK}$ button the handheld device requests product identification information such as product name, manufacturer name, and model type via the serial number on the RFID tag. After that, the operator can see the part identification information on the handheld device. Remanufacturing operators select the information relevant to their work among product identification, usage information, disassembly guidelines, environmental guidelines which are provided by the handheld device. According to the order of the salvage process, an inspection operator selects the usage data viewer and failure-prone parts viewer to check the part condition. Further, parts having the possibility of breakdown are displayed in the failure-prone parts viewer. In this way the operator can perform more accurate inspection than that with the conventional method. The inspection operator marks the parts needed for disposal and recycling by checking on the viewer so that other operators can identify the parts. Based on the inspection information, the disassembly operator selects the disassembly guide showing the process of his/her work. If it is necessary to show the internal side of the product, then DISS shows the assembly sequence of the motor. The recycling operator selects the regulation viewer on the handheld device. This viewer shows the environmental regulations related to the motor. The operator classifies the composition materials of each part according to this guideline and transmits each part to the appropriate next process. Based on the demo scenario of u-PRMS, advantages of the proposed PRMS compared with the current status are summarized in Table 2.

The environmental impact of u-PRMS is calculated by counting the amount of excavator engines remanufactured. The assumptions of the calculation are followings

1) As shown in Table 3, four companies remanufactures their excavators. The market share of the table is used for the calculation. Total market size is considered as 341,000 excavators.

2) If a company remanufactures their products, $37.2 \%$ products can be collected.

3) The barrier of remanufacturing is the lack of the knowledge which can be provided by u-PRMS

The environmental impact of an engine is categorized as 11,600 MJ energy consumption, $860 \mathrm{~kg}$ carbon dioxide emission, $1 \mathrm{~kg}$ carbon monoxide emission, $2.5 \mathrm{~kg}$ nitrogen oxide emission, $4.5 \mathrm{~kg}$ sulfur oxide emission, $1 \mathrm{~kg}$ non-methane hydrocarbons, and $240 \mathrm{~kg}$ solid waste (Smith 2004). Remanufactured excavators were increased by $15 \%$ to $23.5 \%$. Reductions of $12 \%$ were found in carbon dioxide emission and more than $10 \%$ in energy consumption and solid waste. The result of the calculation is shown in Fig. 10. The economic gain of 10 Euros per

Table 3 Market share of excavator manufactures in 2008

\begin{tabular}{ccc}
\hline Company & Market share & Remanufacture \\
\hline A & 18.9 & O \\
\hline B & 10.9 & O \\
\hline C & 5.9 & X \\
\hline D & 5.4 & O \\
\hline E & 5.1 & O \\
\hline F & 4.8 & X \\
\hline G & 3.5 & X \\
\hline H & 3 & X \\
\hline I & 2.9 & X \\
\hline J & 2.9 & X
\end{tabular}

Table 2 Advantages of demonstration scenario compared with the current scenario

\begin{tabular}{|c|c|c|c|}
\hline Task & As-Is scenario & To-Be scenario & Advantages \\
\hline (1)Identification & $\begin{array}{l}\text { Each operator spends time to find the serial } \\
\text { number of individual products and to } \\
\text { search product information }\end{array}$ & $\begin{array}{l}\text { Operator just contacts handheld device to } \\
\text { get radio frequency identification tag of the } \\
\text { product }\end{array}$ & $\begin{array}{l}\text { The time for finding product } \\
\text { information is reduced }\end{array}$ \\
\hline (2)Inspection & $\begin{array}{l}\text { Operator has no information about usage } \\
\text { history of the individual product. Thus, the } \\
\text { operator's experience is the key to } \\
\text { inspection accuracy and the variety of part } \\
\text { to be dealt with }\end{array}$ & $\begin{array}{c}\text { Operator can have usage history } \\
\text { information of the individual product by } \\
\text { PRMS }\end{array}$ & $\begin{array}{l}\text { Even a non-skilled operator can } \\
\text { handle a variety of products in an } \\
\text { accurate fashion }\end{array}$ \\
\hline (3)Disassembly & $\begin{array}{l}\text { In the case of a new and complicated } \\
\text { product in particular, disassembly is not } \\
\text { easy even for the skilled operator }\end{array}$ & $\begin{array}{l}\text { Operator can see the assembly structure } \\
\text { from handheld device with disassembly } \\
\text { sequence provided by the PRMS }\end{array}$ & $\begin{array}{l}\text { Even a non-skilled operator } \\
\text { can perform the disassembly task in } \\
\text { an accurate and optimal fashion (in } \\
\text { the sense of disassembly time) }\end{array}$ \\
\hline (4)Recycling & $\begin{array}{l}\text { Operator sorts out the recyclable/hazardous } \\
\text { part materials based on his own experience, } \\
\text { often resulting in a wrong decision, } \\
\text { especially for a new or unfamiliar material }\end{array}$ & $\begin{array}{l}\text { Operator can identify the material } \\
\text { properties of the part by accessing PRMS } \\
\text { and select appropriate treatment for each } \\
\text { product }\end{array}$ & $\begin{array}{c}\text { The operator can confidently enhance } \\
\text { the rate of recycling while } \\
\text { minimizing the risk of hazard } \\
\text { material }\end{array}$ \\
\hline
\end{tabular}


$\mathrm{kg}$ when calculated versus 1.8 million Euros and scrap metal price of 350 U.S. dollars, you can expect a savings of 16,030,000 U.S. dollars. Economic benefit 1.8 million Euros for carbon dioxide emission reduction and 350 US dollars for metal scrap if carbon dioxide emission certified emission reduction is 10 Euros per $\mathrm{kg}$ and scrap price is 350 dollars per $\mathrm{kg}$. Result findings investigated during the implementation are discussed in the next section.

\section{Discussion}

The prototype implementation shows that the introduction of ubiquitous information engineering for u-PRMS is one of the ways to overcome the trade-off between information level and equipment cost occurring from development complexity. Four modules designed in the system design covered functions required for collecting product information for recovery decision. It is more efficient that the developer concentrates on these modules because system design without predefined basic modules takes a long time. With regard to derivation of data requirement, the data relevant to part condition influences the demand for high-priced equipment such as wireless communication, independent databases, and specialized sensors. Data size and collection frequency are also strongly related to development cost.

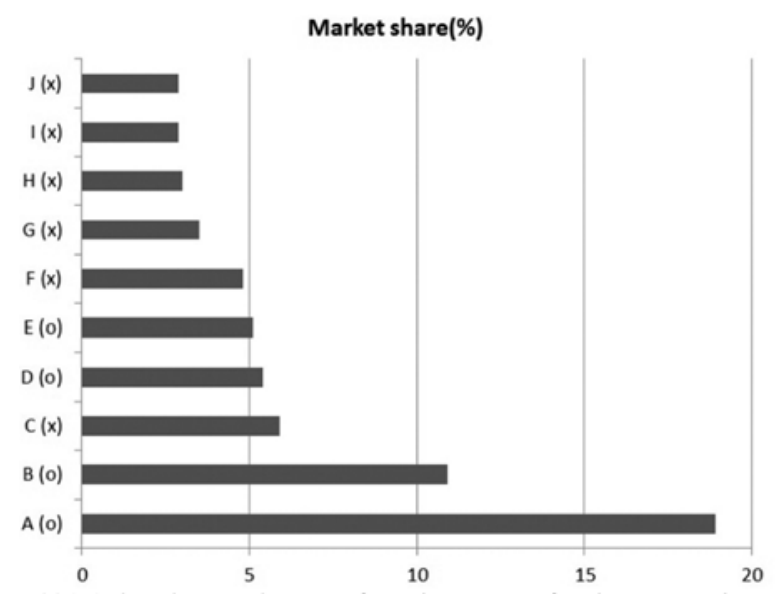

(a) Market share and remanufacturing status of major companies
Therefore, these two factors should be optimized before implementing a system. Furthermore this result means that information content is also as important as information selection mentioned by Kulkarni et al... Development cost can be reduced by using standardized data models defined by ISO 10303 for collecting assembly information, bills of material, and repair history from manufacturers and maintainers. Commercially available modules to handle the standardized data models have already been developed. According to Simon's result equipment cost for implementing specialized devices will decrease. ${ }^{19}$ Recent equipment costs for ubiquitous computing are no longer being reduced. For example, the RFID price of Walmart and the U.S. department of defense did not change for several years. Efficient system design will be a successive breakthrough for introducing PRMS.

Other companies starting to remanufacture results in reduction of environmental impact due to easy to access and use of product information and actual condition. ${ }^{12}$ Through supporting u-PRMS independent companies are able to access the business because they can handle a greater variety of products. Such a system can realize securing of greater quantities of products by independent companies. ${ }^{9,10}$ Tracking systems recording usage history increase the rate of core retrieval more than $0.372 \% .{ }^{4}$ Also, u-PRMS will be applied to other construction machinery as well. Therefore, a greater reduction of environmental impact can be expected from practical application of $\mathrm{u}-$

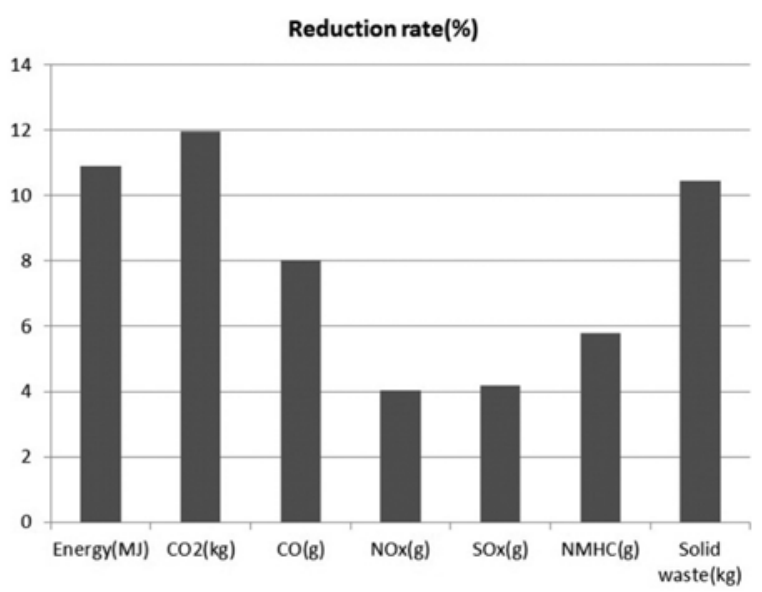

(b) Reduction rate of environment impact

Fig. 10 Analysis results of remanufacturing business

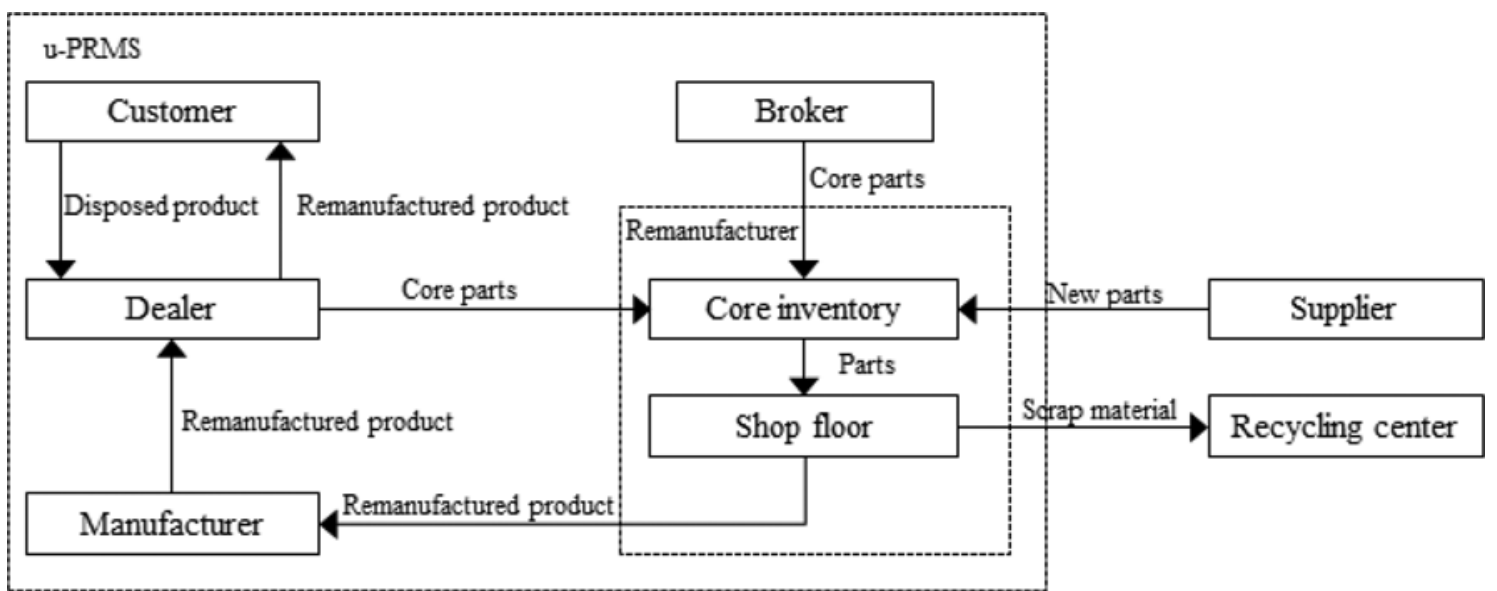

Fig. 11 Material flow of company a remanufacturing construction machineries 
PRMS than the results of this research show. From the perspective of a sustainable society it is worth considering how the developed system can improve the material flow. For such a purpose, let us consider the material flow of company A, remanufacturing construction machinery as shown in Fig. 11. The usefulness of the proposed u-PRMS can be summarized as follows: The remanufacturers can reduce recovery cost and waste by the various decision support functions based on accurate information from the u-PRMS. The errors of the shop floor operator in the salvage process can also be reduced. Further, the Dealer and Broker can determine reusable parts accurately from the usage information from u-PRMS. As well, unnecessary logistic costs caused by wrong decisions by the Dealer and Broker can be eliminated.

\section{Conclusions}

In this paper, the following issues are addressed: 1) the necessity of PRMS, 2) the necessity of ubiquitous computing technology for PRMS, and 3) the necessity of efficient development methods for u-PRMS. In conclusion, the use of ubiquitous computing technology is inevitable for obtaining accurate and live information for products. Then, a generic architecture for u-PRMS was proposed in a previous publication. A key issue in developing u-PRMS in practice is how to design an information acquisition system for u-PRMS. For such a purpose, an information engineering approach called ubiquitous information engineering for designing the implementation architecture is developed.

To show the validity and effectiveness of the developed method, a prototype system has been developed for the remanufacture of a starter motor in construction machinery. Even if it is a prototype, the core functionality required for u-PRMS architecture is fully incorporated through the design process of ubiquitous information engineering. By the proposed ubiquitous information engineering methodology the developers of u-PRMS can reduce efforts developing u-PRMS. Also, the contribution of the u-PRMS to the sustainability of society based on the material flow in the remanufacturing process in practice is explained.

The PRMS itself is not the main concern for the current industry as the remanufacturing market is not very mature. The effort in developing PRMS may be a burden rather than a benefit economically because its existence just increased production cost without increase of product value. Furthermore, developing an advanced PRMS, u-PRMS may be more burdensome for industry except for special products required for constantly monitoring appropriate use and remanufacturing such as military vehicles. As shown, the necessity and effectiveness of u-PRMS is significant so there must be a method for minimizing efforts for developing u-PRMS. In this sense, the ubiquitous information engineering methodology including generic modules that can be used for general products can be a very powerful approach as the u-PRMS for specific products can be developed by adding some customized modules for the specific products under development.

The conclusion based on the research result is as follows. As shown in the development effect of u-PRMS in construction machinery, from the environmental perspective the reduction of energy and solid waste of the whole construction machinery are expected by the application of u-PRMS. The scrap metal is reduced because the return rate of the engine core is increased. The energy consumed by manufacturing the parts from raw material is also reduced. From the industrial perspective the development of u-PRMS via ubiquitous information engineering methodology is shown as one of the methods of applying ubiquitous computing technology to the remanufacturing industry. Future work includes adding factors of remanufacturing market aspects such as the price and demand for each part to the remanufacturing guideline of PRMS and to derive integrated architecture with legacy systems including enterprise resource planning, product lifecycle management, and manufacturing execution system. The future work carried out based on the result contributes application research to enable extension to various types of machinery through the proposed methodology.

\section{ACKNOWLEDGEMENT}

This research was in part supported by the International Research \& Development Program funded by the Ministry of Education, Science and Technology (MEST) of Korea (Grant number: K2100100162110B1300-02910, FY2010), the Eco-Design Graduate School Program funded by Ministry of Environment of Korea, and the Support Program for Graduate School of Engineering Mastership by the Korean Ministry of Knowledge and Economy(MKE).

\section{REFERENCES}

1. Thierry, M., Salomon, M., Van Nunen, J., and Van Wassenhove, L., "Strategic Issues in Product Recovery Management," California Management Review, Vol. 37, No. 2, pp. 114-135, 1995.

2. Gungor, A. and Gupta, S. M., "Issues in Environmentally Conscious Manufacturing and Product Recovery: A Survey," Computers \& Industrial Engineering, Vol. 36, No. 4, pp. 811-853, 1999.

3. Kerr, W. and Ryan, C., "Eco-Efficiency Gains from Remanufacturing: A Case Study of Photocopier Remanufacturing at Fuji Xerox Australia," Journal of Cleaner Production, Vol. 9, No. 1, pp. 75-81, 2001.

4. Smith, V. M. and Keoleian, G. A., "The Value of Remanufactured Engines: Life-Cycle Environmental and Economic Perspectives," Journal of Industrial Ecology, Vol. 8, No. 1-2, pp. 193-221, 2004.

5. Lee, G. B. and Badrul, O., "Integrating Axiomatic Design Principles into Sustainable Product Development," Int. J. Precis. Eng. Manuf.Green Tech., Vol. 1, No. 2, pp. 107-117, 2014.

6. Matsumoto, M., "Business Frameworks for Sustainable Society: A Case Study on Reuse Industries in Japan," Journal of Cleaner Production, Vol. 17, No. 17, pp. 1547-1555, 2009.

7. Matsumoto, M., "Development of a Simulation Model for Reuse Businesses and Case Studies in Japan," Journal of Cleaner Production, Vol. 18, No. 13, pp. 1284-1299, 2010.

8. Östlin, J., Sundin, E., and Björkman, M., "Importance of ClosedLoop Supply Chain Relationships for Product Remanufacturing," 
International Journal of Production Economics, Vol. 115, No. 2, pp. 336-348, 2008.

9. Geyer, R. and Jackson, T., "Supply Loops and Their Constraints: The Industrial Ecology of Recycling and Reuse," California Management Review, Vol. 46, No. 2, pp. 55-73, 2004.

10. Sundin, E., Tang, O., and Mårtén, E., "The Swedish Remanufacturing Industry: An Overview of Present Status and Future Potential," Proc. of the International CIRP Life Cycle Engineering Seminar, Paper No. BM4 on the LCE-05 CD, 2005.

11. Ilgin, M. A. and Gupta, S. M., "Recovery of Sensor Embedded Washing Machines using a Multi-Kanban Controlled Disassembly Line," Robotics and Computer-Integrated Manufacturing, Vol. 27, No. 2, pp. 318-334, 2011.

12. Luttropp, C. and Johansson, J., "Improved Recycling with Life Cycle Information Tagged to the Product," Journal of Cleaner Production, Vol. 18, No. 4, pp. 346-354, 2010.

13. Ondemir, O., Ilgin, M. A., and Gupta, S. M., "Optimal End-of-Life Management in Closed-Loop Supply Chains using RFID and Sensors," IEEE Transactions on Industrial Informatics, Vol. 8, No. 3, pp. 719-728, 2012.

14. Thomas, V., Neckel, W., and Wagner, S., "Information Technology and Product Lifecycle Management," Proc. of the IEEE International Symposium on Electronics and the Environment, pp. 54-57, 1999 .

15. Um, J., Yoon, J.-S., and Suh, S.-H., "An Architecture Design with Data Model for Product Recovery Management Systems," Resources, Conservation and Recycling, Vol. 52, No. 10, pp. 11751184, 2008.

16. Krikke, H., Van Harten, A., and Schuur, P., "On a Medium Term Product Recovery and Disposal Strategy for Durable Assembly Products," International Journal of Production Research, Vol. 36, No. 1, pp. 111-140, 1998.

17. White, C. D., Masanet, E., Rosen, C. M., and Beckman, S. L., "Product Recovery with Some Byte: An Overview of Management Challenges and Environmental Consequences in Reverse Manufacturing for the Computer Industry," Journal of Cleaner Production, Vol. 11, No. 4, pp. 445-458, 2003.

18. Lambert, A. J., "Disassembly Sequencing: A Survey,” International Journal of Production Research, Vol. 41, No. 16, pp. 3721-3759, 2003.

19. Simon, M., Bee, G., Moor, P., Pu, J.-S., and Xie, C., "Modeling of the Life Cycle of Products with Data Acquisition Features," Computers in Industry, Vol. 45, pp. 111-122, 2001.

20. Kulkarni, A., Parlikad, A., McFarlane, D., and Harrison, M., "Networked RFID Systems in Product Recovery Management," Proc. of the IEEE International Symposium on Electronics and the Environment, pp. 66-71, 2005.

21. McFarlane, D., Sarma, S., Chirn, J. L., Wong, C., and Ashton, K.,
"Auto ID Systems and Intelligent Manufacturing Control," Engineering Applications of Artificial Intelligence, Vol. 16, No. 4, pp. 365-376, 2003.

22. Kiritsis, D., Bufardi, A., and Xirouchakis, P., "Research Issues on Product Lifecycle Management and Information Tracking using Smart Embedded Systems," Advanced Engineering Informatics, Vol. 17, No. 3, pp. 189-202, 2003.

23. Moor, P. R., "Environmental Life Cycle Information Management and Acquisition for Consumer Products," http://www.mrg.dmu.ac. uk/elima/elima (Accessed 29 May 2006)

24. Suh, S.-H., Shin, S.-J., Yoon, J.-S., and Um, J.-M., "UbiDM: A New Paradigm for Product Design and Manufacturing via Ubiquitous Computing Technology," International Journal of Computer Integrated Manufacturing, Vol. 21, No. 5, pp. 540-549, 2008.

25. Yoon, J.-S., Shin, S.-J., and Suh, S.-H., “A Conceptual Framework for the Ubiquitous Factory," International Journal of Production Research, Vol. 50, No. 8, pp. 2174-2189, 2012.

26. Lee, B.-E. and Suh, S.-H., "An Architecture for Ubiquitous Product Life Cycle Support System and Its Extension to Machine Tools with Product Data Model," The International Journal of Advanced Manufacturing Technology, Vol. 42, No. 5-6, pp. 606-620, 2009.

27. Jeong, S., Hur, S. M., and Suh, S.-H., "A Conceptual Framework for Computer-Aided Ubiquitous System Engineering: Architecture and Prototype," International Journal of Computer Integrated Manufacturing, Vol. 22, No. 7, pp. 671-685, 2009.

28. Cha, J.-M. and Suh, S.-H., "Developing a Conceptual Framework for UT Based LCA," in: Glocalized Solutions for Sustainability in Manufacturing, Jürgen, H. and Christoph, H., (Eds.), Springer, pp. 587-592, 2011.

29. Hatcher, G. D., Ijomah, W., and Windmill, J., "Integrating Design for Remanufacture into the Design Process: The Operational Factors," Journal of Cleaner Production, Vol. 39, pp. 200-208, 2013.

30. ISO No. 14649-1, "Industrial Automation Systems and Integration, Physical Device Control - Data Model for Computerized Numerical Controllers - Part 1: Overview and Fundamental Principles," 2003.

31. ISO No. 10303-403, "Industrial Automation Systems and Integration - Product Data Representation and Exchange - Part 403: Application Module: AP203 Configuration Controlled 3D Design of Mechanical Parts and Assemblies,” 2010.

32. ISO No. 10303-239, "Industrial Automation Systems and Integration - Product Data Representation and Exchange - Part 239: Application Protocol: Product Life Cycle Support,” 2012.

33. Chiu, M.-C. and Teng, L.-W., "Sustainable Product and Supply Chain Design Decisions under Uncertainties," Int. J. Precis. Eng. Manuf., Vol. 14, No. 11, pp. 1953-1960, 2013.

34. Sundin, E. and Bras, B., "Making Functional Sales Environmentally and Economically Beneficial through Product Remanufacturing," Journal of Cleaner Production, Vol. 13, No. 9, pp. 913-925, 2005. 
35. Cha, J.-M. and Suh, S.-H., "Conceptual Model for UT-Based Life Cycle Assessment (u-LCA)," Proc. of the $9^{\text {th }}$ International Conference on EcoBalance, pp. 408-411, 2010.

36. Leonard, J., "Systems Engineering Fundamentals," Defense Acquisition University Press, 2001.

37. Einsporn, T., "eCl@ss - The Leading Classification System," Cologne Institute for Business Research Consult Ltd., 2005.

38. Bitner, M. J., Ostrom, A. L., and Morgan, F. N., "Service Blueprinting: A Practical Technique for Service Innovation," California Management Review, Vol. 50, No. 3, pp. 1-24, 2008.

39. Cross, N., "Engineering Design Method," Wiley, $4^{\text {th }}$ Ed., 2000.

40. Hauser, J. R. and Clausing, D., "The House of Quality," Harvard Business Review, Vol. May-June, pp. 63-73, 1988.

41. Jun, H.-B., Cusin, M., Kiritsis, D., and Xirouchakis, P., “A MultiObjective Evolutionary Algorithm for EOL Product Recovery Optimization: Turbocharger Case Study," International Journal of Production Research, Vol. 45, No. 18-19, pp. 4573-4594, 2007.

42. ISO No. 10303-224 "Industrial Automation Systems and Integration - Product Data Representation and Exchange - Part 224: Application Protocol: Mechanical Product Definition for Process Planning using Machining Features," 2006. 